\title{
Zur Quantentheorie der gewinkelten Valenz, I. Mitteilung: Eigenfunktion ụnd Valenzbetätigung des Zentralatoms.
}

\author{
Von Kurt Artmann \\ Aus dem Institut für Theoretische Physik der Universität Hamburg
}

(Z. Naturforschg. 1, 426-432 [1946]; eingegangen am 26. Juni 1946)

\begin{abstract}
Die Slater-Paulingsche Theorie der gewinkelten Valenz (= Tatsache, daß mehratomige Moleküle wie $\mathrm{NH}_{3}$ Pyramidenform und $\mathrm{CH}_{4}$ Tetraederform besitzen) wird in der Richtung vervollständigt, daß das Zentralatom wesentlich als Vielelektronenproblem behandelt wird. (Die Behandlung des Moleküls soll in der II. Mitteilg. erfolgen.) Es wird in schlüssigerer Weise als bei Slater nachgewiesen, daß die gewinkelte Valenz eine Eigenschaft ist, die dem Zentralatom bereits innewohnt. D. h. es wird gezeigt, daß z. B. die Aufenthaltswahrscheinlichkeit der drei Valenzelektronen des Stickstoffs am größten in drei senkrecht aufeinander stehenden Richtungen ist, und die Aufenthaltswahrscheinlichkeit der vier Valenzelektronen des Kohlenstoffs am größten in vier Richtungen ist, die nach den vier Ecken eines regulären Tetraeders weisen.

In diesem Zusammenhang wird abweichend von $\mathrm{H} \ddot{\mathrm{u}} \mathrm{ckel}$ die Stabilität des Benzolrings nicht durch die sechs Elektronen erklärt, die nicht an einzelnen Atomen lokalisierbar sind, sondern durch die (gewinkelte) Valenzbetätigung des dreiwertigen Kohlenstoffatoms, dessen Valenzarme vom Mittelpunkt eines gleichseitigen Dreiecks aus nach dessen Ecken gerichtet sind.
\end{abstract}

1. Problemstellung. Eine Reihe von mehratomigen Molekülen besitzt die Eigenschaft, daß ihre Atome nicht auf einer Geraden liegen, sondern von einem Zentralatom aus gesehen, unter gewissen Winkeln angeordnet sind. Die typischsten Beispiele sind $\mathrm{H}_{2} \mathrm{O}, \mathrm{NH}_{3}, \mathrm{CH}_{4}$. Das Sauerstoffatom des Wassers bindet die beiden Wasserstoffatome unter einem Winkel von ungefähr $110^{\circ}$; die drei H-Atome des $\mathrm{NH}_{3}$ lagern sich an das N-Atom in drei Richtungen an, die (näherungsweise) aufeinander senkrecht stehen; und beim $\mathrm{CH}_{4}$ bilden die vier H-Atome die Ecken eines regulären Tetraeders mit dem C-Atom als Mittelpunkt.

Diese sogenannte gewinkelte Valenz tritt nach der Theorie von J. C. Slater ${ }^{1}$ und L. Pauling ${ }^{2}$ immer dann ein, wenn $p$-Elektronen des Zentralatoms an der Bindung beteiligt sind. Das sind Elektronen, deren Eigenfunktion nach Slate $\mathrm{r}^{\mathbf{1}}$ in der Form

$$
\psi_{p}=\left(\mathfrak{a} \mathfrak{r}^{0}\right) \cdot f(r)
$$

dargestellt werden. Hierbei ist $\mathfrak{r}=\mathfrak{r}^{0} \cdot r\left(\left|\mathfrak{r}^{0}\right|=1\right.$, $|\mathfrak{r}|=r)$ der Ortsvektor des Elektrons und a ein mit dem Atomkern fest verbunden gedachter (von $\mathfrak{r}$ un-

1 Physic. Rev. 37, 481 [1931]; 38, 1109 [1931].

2 J. Amer. chem. Soc. 531367 [1931]; Physic. Rev. 37, 1185 [1931]. abhängiger) Einheitsvektor. Die ( ) bedeutet das skalare Produkt der (Einheits-) Vektoren $\mathfrak{a}$ und $\mathfrak{r}^{0}$. Bei der gewinkelten Valenz können auch $s$-Elektronen beteiligt sein, deren Eigenfunktion bekanntlich die Gestalt

$$
\psi_{s}=g(r)
$$

besitzt, wobei $g(r)$ wie $f(r)$ zum Atomrand $(r \rightarrow \infty)$ exponentiell abklingen.

Um auch dem mit der Literatur weniger vertrauten Leser einen möglichst raschen Einblick in den hier angeschnittenen Problemkreis zu ermöglichen, soll zunächst die Theorie des Verfassers entwickelt werden, ohne auf frühere Arbeiten anderer Autoren eingehend Bezug zu nehmen. Dies wird in aller Ausführlichkeit erst in der III. Mitteilg. geschehen. Nur am Beispiel des Sauerstoffs soll schon hier der Unterschied zwischen der Theorie des Verfassers und der Slater-Pauling schen Auffassung in groben Zügen skizziert werden.

Die Grundlage dieser Arbeit bildet die Slatersche Theorie des Atom- und Molekülbaus, die jedoch in etwas anderer Weise ausgebaut wird*, als es bei Slater geschieht. Hierdurch wird der bereits von Slater und Pauling vertretene

* Vergl. hierzu die Schlußbemerkung dieser Mitteilung. 
Grundgedanke, daß die gewinkelte Valenz eine Eigenschaft ist, die dem Zentralatom (O, N, bzw. C) bereits innewohnt, und nicht von der Art der zu bindenden Atome abhängt, bedeutend ausgeprägter als bei Slater und Pauling zum Ausdruck kommen.

In diesem Zusammenhang ist es besonders einfach verständlich, warum der Benzolring die Form eines ebenen Sechsecks besitzt. Dieses Problem ist zwar schon von E. Hü ckel ${ }^{3}$ behandelt worden. Da aber hier eine andere Begründung für die besonders große Stabilität des ebenen Sechser-Rings gegeben wird als von $\mathrm{Hückel}$, so ist dieses Problem im Zusammenhang mit der gewinkelten Valenz noch einmal aufgegriffen worden.

2. Die Hei sen berg-Slater-Determinante. Zu einem raschen Verständnis für die gewinkelte Valenz gelangt man schon, wenn man die Eigenfunktion des Zentralatoms (O, N, bzw. C) berechnet. Hierbei beschränken wir uns darauf, die Eigenfunktion der Valenz-Elektronen aufzustellen. Das sind diejenigen Elektronen, deren Spin nicht schon durch den entgegengesetzt gerichteten Spin eines anderen Elektrons des Zentralatoms kompensiert (abgesättigt) wurde. Diese Valenzelektronen haben demnach parallelen Spin. Ihre Anzahl $n$ ist die Wertigkeit des betreffenden Atoms. Die übrigen (abgesättigten) Elektronen des Zentralatoms denkt man sich kugelsymmetrisch um den Kern verschmiert. Sie bilden zusammen mit dem Kern das Rumpfpotential.

Wir vernachlässigen die Coulombsche Wechselwirkung zwischen den Valenzelektronen. Wegen des Pauli-Prinzips, das Antisymmetrie der Eigenfunktion bei Vertauschung zweier Elektronen verlangt, läßt sich dann die Eigenfunktion unseres Zentralatoms in Form einer Determinante darstellen. Bezeichnen $\varphi_{k}(k=1, \ldots n)$ die $n$ Eigenfunktionen der $n$ Valenzelektronen und bezeichne das an irgendeiner dieser Funktionen $\varphi_{k}$ angebrachte Argument $i(1<i \ll n)$ die Nummer des . Elektrons: $\varphi_{k}(i)$, so lautet die HeisenbergSlate r-Determinante:

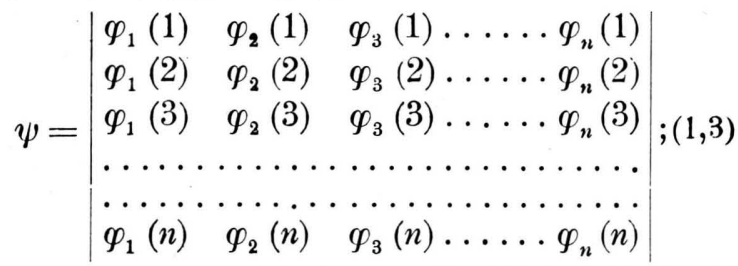

${ }^{3}$ Z. Physik 70, 204 [1931]; 76, 628 [1932]; 83, 632 [1933]. $\psi$ hängt also von den $n$ Ortsvektoren $\mathfrak{r}_{1}, \mathfrak{r}_{2}, \ldots \mathfrak{r}_{n}$ der $n$ Valenzelektronen ab.

Nun ist $|\psi|^{2}$ proportional der Aufenthaltswahrscheinlichkeit des Elektrons $i$ an der Stelle $\mathfrak{r}$. Andererseits besteht nach W.Heitler und F.London ${ }^{4}$ das Wesen der chemischen Bindung zwischen zwei Atomen darin, daß die Valenzelektronen von einem Atom zum anderen übertreten können und daher dauernd ihre Plätze auswechseln. Wie bereits Slater bemerkte, leuchtet es ein, daß sich einwertige Atome an unser Zentralatom in denjenigen Richtungen anlagern werden, in welchen dieser Platzwechsel am häufigsten auftreten kann. Das sind die Richtungen, in welchen die Aufenthaltswahrscheinlichkeit $|\psi|^{2}$ der Valenzelektronen des Zentralatoms am größten ist. Zur strengen Begründung dieses Gedankengangs wird in der II. Mitteilg. gezeigt werden, daß die Energie des Moleküls in diesem Fall den tiefsten Wert hat.

Aufgabe dieser Mitteilung ist es daher, das Ergebnis dieser Energieberechnung in möglichst einfacher Weise zu illustrieren. Das geschieht am zweckmäßigsten dadurch, daß man in den $\varphi_{k}(i)$ von $(1,3)$ die Beträge $\boldsymbol{r}_{\boldsymbol{i}}$ der Ortsvektoren, die ja unabhängig voneinander beliebige Werte annehmen können, willkürlich einander gleich setzt, und dann diejenigen Richtungen $\mathfrak{r}_{i}{ }^{0}$ der Ortsvektoren der Valenzelektronen aufsucht, bei welcher die Determinante $(1,3)$ ihren größten Wert annimmt. In diesen Richtungen werden sich bei der Molekülbildung einwertige Atome an unser Zentralatom anlagern, wenn die Wechselwirkung zwischen den gebundenen Atomen zunächst vernachlässigt wird. Wir beschränken uns hierbei auf die Fälle, daß die Valenzelektronen $s$ - oder $p$-Elektronen sind, d. h. wir setzen für die $\varphi_{k}(i)$ in $(1,3)$ die Darstellung $(1,2)$ bzw. $(1,1)$ der $s$ - bzw. $p$-Funktionen ein. Eine Erweiterung der Theorie auf $d$-Elektronen ist im Prinzip zwar möglich. Da sich dann aber die Determinante $(1,3)$ nicht mehr in so einfacher Weise auswerten läßt, wie es bei den $s$ - und $p$-Elektronen der Fall sein wird, so sehen wir von dieser Erweiterung der Theorie ab, zumal die für die Praxis wichtigsten Atome nur $s$ - und $p$-Elektronen als Valenzelektronen besitzen.

3. Die Eigenfunktion $\psi_{p p}$. Wir beginnen mit der Berechnung der Determinante $(1,3)$ für den Fall des Sauerstoffs, Hier ist $n=2$, und beide Valenzelektronen sind $p$-Elektronen. Deshalb wird die Eigenfunktion mit $\psi_{p p}$ bezeichnet. Die willkürlich 4 Z. Physik 44, 455 [1927]. 
annehmbaren (mit dem Atomkern fest verbunden gedachten) Achsenvektoren mögen $\mathfrak{a}$ und $\mathfrak{b}$ genannt werden. Dann lautet die Sauerstoff-Eigenfunktion nach $(1,1),(1,3)$ :

$$
\left.\begin{array}{rl}
\psi_{p p} & =\left|\begin{array}{ll}
\left(\mathfrak{a} \mathfrak{r}_{1}{ }^{0}\right) \cdot f\left(r_{1}\right) & \left(\mathfrak{b} \mathfrak{r}_{1}{ }^{0}\right) \cdot f\left(r_{1}\right) \\
\left(\mathfrak{a} \mathfrak{r}_{2}{ }^{0}\right) \cdot f\left(r_{2}\right) & \left(\mathfrak{b} \mathfrak{r}_{2}{ }^{0}\right) \cdot f\left(r_{2}\right)
\end{array}\right| \\
& =f\left(r_{1}\right) \cdot f\left(r_{2}\right) \cdot\left\{\left(\mathfrak{a} \mathfrak{r}_{1}{ }^{0}\right)\left(\mathfrak{b} \mathfrak{r}_{2}{ }^{0}\right)-\left(\mathfrak{a} \mathfrak{r}_{2}{ }^{0}\right)\left(\mathfrak{b} \mathfrak{r}_{1}{ }^{0}\right)\right\}
\end{array}\right\}(1,4)
$$

Wir führen nun in $(1,4)$ für das Vektorenprodukt $[\mathfrak{a} \mathfrak{b}]$ die Abkürzung $\mathfrak{c}$ ein und setzen ferner $\mathfrak{c}=c \cdot c^{0}$ mit $\left|\mathfrak{c}^{0}\right|=1$. Dann ist $c=|\mathfrak{c}|=|[\mathfrak{a} \mathfrak{b}]|=$ $\sin \gamma$, wenn $\gamma$ der Winkel ist, den die Achsenvektoren $\mathfrak{a}$ und $\mathfrak{b}$ miteinander bilden. Dann geht $(1,4)$ über in

$$
\psi_{p p}=\sin \gamma \cdot f\left(r_{1}\right) \cdot\left(f r_{2}\right)\left(\mathfrak{c}^{0}\left[\mathfrak{r}_{1}{ }^{0} \mathfrak{r}_{2}{ }^{0}\right]\right) . \quad\left(1,4^{\prime}\right)
$$

Der Winkel $\gamma$ der beiden Achsenvektoren $\mathfrak{a}$ und $\mathfrak{b}$ geht in $\left(1,4^{\prime}\right)$ nur in Form des konstanten Faktors $\sin \gamma$ ein. Nun ist nur die auf 1 normierte Eigenfunktion von Interesse. Bei dieser Normierung fällt der Faktor sin $\gamma$ heraus, so daß $\psi$ (nach Normierung) vom Winkel $\gamma$ der beiden Achsenvektoren $\mathfrak{a}$ und $\mathfrak{b}$ unabhängig ist.

Wir denken uns die beiden Valenzelektronen des Sauerstoffs auf einer festen Kugel $r_{1}=r_{2}=r$ beweglich. Dann hat die Aufenthaltswahrscheinlichkeit $\left|\psi_{p p}\right|^{2}$ dann ihren größten Wert, wenn das skalarvektorielle Produkt $\left(\mathfrak{c}^{0}\left[\mathfrak{r}_{1}{ }^{0} \mathfrak{r}_{2}{ }^{0}\right]\right)$ seinen größten Wert annimmt. Da $\left(\mathfrak{c}^{0}\left[\mathfrak{r}_{1}{ }^{0} \mathfrak{r}_{2}{ }^{0}\right]\right)$ gleich dem Volumen des Parallelepipeds ist, das von den Einheitsvektoren $\mathfrak{c}^{0}, \mathfrak{r}_{1}{ }^{0}, \mathfrak{r}_{2}{ }^{0}$ gebildet wird, so hat $\left(\mathfrak{c}^{0}\left[\mathfrak{r}_{1}{ }^{0} \mathfrak{r}_{2}{ }^{0}\right]\right)$ seinen größten Wert, wenn diese drei Einheitsvektoren senkrecht aufeinander stehen, speziell also der Ortsvektor $\mathfrak{r}_{1}=r_{1} \cdot \mathrm{r}_{1}{ }^{0}$ des 1 . Valenzelektrons senkrecht auf dem Ortsvektor $\mathfrak{r}_{2}=$ $r_{2} \cdot \dot{\mathfrak{x}}_{2}{ }^{0}$ des 2. Valenzelektrons steht. Es ist daher plausibel, daß das $\mathrm{O}$-Atom im $\mathrm{H}_{2} \mathrm{O}$ zwei $\mathrm{H}$-Atome unter rechtem Winkel bindet, wenn die Wechselwirkung zwischen den beiden $\mathrm{H}$-Atomen vernachlässigt wird.

Da eine chemische Bindung zwischen zwei Atomen nach Heitler und London nur durch Elektronen mit antiparallelem Spin bewirkt wird, so müssen die Elektronen der beiden vom Sauerstoff gebundenen Wasserstoffatome einen Spin besitzen, der antiparallel dem Spin der beiden (spingleichen) Valenzelektronen des Sauerstoffs ist. Es müssen also auch die Elektronen der beiden H-Atome spingleich sein. Zwei solche Atome stoßen sich aber nach der Heitler-London- schen Theorie ab. Die Wechselwirkung zwischen den beiden $\mathrm{H}$-Atomen ist also bestrebt, den rechten Winkel, unter dem die beiden H-Atome bei Vernachlässigung ihrer gegenseitigen Wechselwirkung am O-Atom gebunden wären, zu vergrößern. Auf diese Weise ist es verständlich, daß der beobachtete Valenzwinkel am Sauerstoff, nicht genau $90^{\circ}$, sondern $110^{\circ}$ beträgt. Entsprechend ist die gewinkelte Form von $\mathrm{H}_{2} \mathrm{~S}$ zu verstehen, weil S genau wie $\mathrm{O}$ zwei $p$-Elektronen als Valenzelektronen besitzt.

4. Der Unterschied gegenüber der Slater-Paulingschen Theorie. Während bei uns die gewinkelte Valenzbetätigung des O-Atoms darauf zurückgeführt wird, daß der Orts-Vektor $\mathfrak{r}_{1}$ des 1 . Valenzelektrons mit größter Wahrscheinlichkeit senkrecht auf dem Orts-Vektor $\mathfrak{r}_{2}$ des 2. Valenzelektrons steht, soll dagegen nach Slater und $\mathrm{Pau}$ ling die gewinkelte Valenzbetätigung des Sauerstoffatoms darauf beruhen, daß die Achsen-Veltoren $\mathfrak{a}$ und $\mathfrak{b}$ der $p$-Funktionen senkrecht aufeinander stehen müssen. Diese Orthogonalitätseigenschaft der Achsenvektoren $\mathfrak{a}$ und $\mathfrak{b}$ ist nach $\mathrm{S}$ la te $\mathrm{r}$ und Pauling eine Folge derTatsache, daßirgend zwei Eigenfunktionen des Zentralatoms - im Falle des Sauerstoffs die Funktionen $\left(\mathfrak{a r}^{0}\right) \cdot f(r)$ und $\left(\mathfrak{b r}{ }^{0}\right) \cdot f(r)$ - orthogonal sein müssen. D. h. $\int_{-\infty}^{+\infty}\left(\mathfrak{a} \mathfrak{r}^{0}\right)\left(\mathfrak{b} \mathfrak{r}^{0}\right) \cdot f^{2}(r) d x d y d z=0$, woraus $\mathfrak{a} \perp \mathfrak{b}$ folgt. Da aber in unserer Theorie der Winkel $\gamma$ der Achsenvektoren $\mathfrak{a}$ und $\mathfrak{b}$ nicht mehr vorkommt, so kann im Rahmen unserer Theorie aus der Orthogonalitätsforderung $\mathfrak{a} \perp \mathfrak{b}$ nichts für die gewinkelte Valenz des Sauerstoffatoms folgen. Das ist der wesentliche Punkt, in dem wir uns von Slater und Pauling unterscheiden.

5. Die Eigenfunktion $\psi_{s p}$. Wir gehen nunmehr $\mathrm{zu}$ einem zweiwertigen Atom über, dessen eines Valenzelektron ein $s$ - und dessen anderes Valenzelektron ein $p$-Elektron ist. Die zugehörige Eigenfunktion, die mit $\psi_{s p}$ bezeichnet werden soll, lautet nach $(1,1),(1,2),(1,3)$ : 


$$
\left.\begin{array}{rl}
\psi_{s p} & =\left|\begin{array}{ll}
g\left(r_{1}\right) & \left(\mathfrak{a} \mathfrak{r}_{1}{ }^{0}\right) f\left(r_{1}\right) \\
g\left(r_{2}\right) & \left(\mathfrak{a} \mathfrak{r}_{2}{ }^{0}\right) f\left(r_{2}\right)
\end{array}\right| \\
& =g\left(r_{1}\right) \cdot f\left(r_{2}\right)\left(\mathfrak{a} \mathfrak{r}_{2}{ }^{0}\right)-g\left(r_{2}\right) \cdot f\left(r_{1}\right) \cdot\left(\mathfrak{a} \mathfrak{r}_{1}{ }^{0}\right) .
\end{array}\right\}
$$

Denken wir uns auch hier die beiden Valenzelektronen auf einer festen Kugel $r_{1}=r_{2}=r$ beweglich, so nimmt $(1,5)$ die Gestalt an

$$
\psi_{s p}=g(r) \cdot f(r)\left(\mathfrak{a}, \mathfrak{r}_{2}{ }^{0}-\mathfrak{r}_{1}{ }^{0}\right)
$$

Die Aufenthaltswahrscheinlichkeit $\left|\psi_{s p}\right|^{2}$ hat demnach ihren größten Wert, wenn das skalare Produkt $\left(\mathfrak{a}, \mathfrak{r}_{2}{ }^{0}-\mathfrak{r}_{1}{ }^{0}\right)$ seinen größten Wert hat, d. h. wenn $\mathfrak{r}_{1}{ }^{0}=-\mathfrak{r}_{2}{ }^{0}(= \pm \mathfrak{a})$ ist. Ein solches Atom müßte daher zwei einwertige Atome in gestreckter Form binden. Die Wechselwirkung zwischen den beiden gebundenen Atomen ändert an der gestreckten Form dieses Moleküls nichts mehr.

Auf diese Weise ist die gestreckte Form von $\mathrm{HgCl}_{2}, \mathrm{HgBr}_{2}, \mathrm{HgJ}_{2} \mathrm{zu}$ verstehen. Das isolierte $\mathrm{Hg}$-Atom besitzt nämlich in der äußersten $(P-)$ Schale zwei $s$-Elektronen mit entgegengesetztem Spin, wäre also zunächst als nullwertịg anzusehen. $\mathrm{Da}$ aber $\mathrm{Hg}$ in den oben genannten Molekülen erfahrungsgemäß zweiwertig auftritt, so müssen in diesen Verbindungen die beiden äußeren Elektronen des Hg-Atoms parallelen Spin besitzen. Da sich nach dem Pauli-Prinzip nicht zwei Elektronen mit parallelem Spin in einer $s$-Bahn aufhalten können [die zugehörige Eigenfunktion $\psi_{s e}$ wäre nach $(1,2),(1,3)$ identisch Null], so muß man annehmen, daß bei der Bildung des $\mathrm{HgCl}_{2}$ Moleküls infolge innermolekularer Kräfte eines der beiden $s$-Elektronen unter Spinumkehr aus der $s$-Bahn herausgebracht wird. Es ist naheliegend anzunehmen, daß dieses Elektron in eine $p$-Bahn gelangt, so daß das Hg-Atom im Molekülverband ein $s$ - und ein $p$-Elektron als Valenzelektronen besitzt. Das Hg-Atom besitzt dahèr (im Molekülverband) die Eigenfunktion $(1,5)$ und bindet daher zwei einwertige Atome in gestreckter Form.

Nach dieser Auffassung müßten auch die Erdalkalien zwei einwertige Atome in gestreckter Form binden. Denn das einzelne Erdalkali-Atom besitzt genau wie das einzelne $\mathrm{Hg}$-Atom in der äußersten Schale zwei (abgesättigte) $s$-Elektronen, tritt aber im Molekülverband zweiwertig auf. Man. muß daher annehmen, daß die Erdalkalien sich bei der Molekülbildung wie $\mathrm{Hg}$ verhalten. Über den experimentellen Befund ist dem Verfasser allerdings nichts bekannt.

6. Die Eigenfunktion $\psi_{p p p}$. Gehen wir jetzt zum Stickstoff über, so liegt hier der Fall vor, daß drei Valenzelektronen in $p$-Bahnen gebunden sind. Ihre willkürlich zu denkenden Achsenrichtungen mögen $\mathfrak{a}, \mathfrak{b}, \mathfrak{c}$ genannt werden. Nach $(1,1),(1,3)$ lautet daher die Stickstoff-Eigenfunktion

$$
\psi_{p p p}=\left|\begin{array}{lll}
\left(\mathfrak{a} \mathfrak{r}_{1}{ }^{0}\right) \cdot f\left(r_{1}\right) & \left(\mathfrak{b} \mathfrak{r}_{1}{ }^{0}\right) \cdot f\left(r_{1}\right) & \left(\mathfrak{c} \mathfrak{r}_{1}{ }^{0}\right) \cdot f\left(r_{1}\right) \\
\left(\mathfrak{a} \mathfrak{r}_{2}{ }^{0}\right) \cdot f\left(r_{2}\right) & \left(\mathfrak{b} \mathfrak{r}_{2}{ }^{0}\right) \cdot f\left(r_{2}\right) & \left(\mathfrak{c} \mathfrak{r}_{2}{ }^{0}\right) \cdot f\left(r_{2}\right) \\
\left(\mathfrak{a} \mathfrak{r}_{3}{ }^{0}\right) \cdot f\left(r_{3}\right) & \left(\mathfrak{b} \mathfrak{r}_{3}{ }^{0}\right) \cdot f\left(r_{3}\right) & \left(\mathfrak{c} \mathfrak{r}_{3}{ }^{0}\right) \cdot f\left(r_{3}\right)
\end{array}\right| \cdot(1,6)
$$

Nach einem bekannten Satz der Determinantentheorie läßt sich $\psi_{p p p}$ in der Form schreiben

$\psi_{p p p}=(\mathfrak{a}[\mathfrak{b} \mathfrak{c}]) \cdot\left(\mathfrak{r}_{1}{ }^{0}\left[\mathfrak{r}_{2}{ }^{0} \mathfrak{r}_{3}{ }^{0}\right]\right) \cdot f\left(r_{1}\right) \cdot f\left(r_{2}\right) \cdot f\left(r_{3}\right)$.

Hierbei ist $\left(\mathfrak{r}_{1}{ }^{0}\left[\mathrm{r}_{2}{ }^{0} \mathfrak{r}_{3}{ }^{0}\right]\right)$ gleich dem Volumen des aus den drei Einheitsvektoren $\mathfrak{r}_{1}{ }^{0}, \mathfrak{r}_{2}{ }^{0}, \mathfrak{r}_{3}{ }^{0}$ gebildeten Parallelepipeds. Entsprechendes gilt für das skalar-vektorielle Produkt $(\mathfrak{a}[\mathfrak{b} \mathfrak{c}])$. Nun ist aber auch hier nur die auf 1 normierte Eigenfunktion $\psi_{p p p}$ von Interesse. Da $\psi_{p p p}$ von den Achsenrichtungen nur in Form eines konstanten Faktors $(\mathfrak{a}[\mathfrak{b} c])$ abhängt, so ist $\psi_{p p p}$ nach der Normierung von den drei (mit dem Kern fest verbunden gedachten) Achsenrichtungen $\mathfrak{a}, \mathfrak{b}, \mathfrak{c}$ unabhängig.

Bei festem Abstand $r_{1}=r_{2}=r_{3}=r$ hat die Auf- enthaltswahrscheinlichkeit $\left|\psi_{p p p}\right|^{2}$ dannihren grö $\beta$ ten Wert, wenn das Volumen $\left(\mathfrak{r}_{1}{ }^{0}\left[\mathrm{r}_{2}{ }^{0} \mathfrak{r}_{3}{ }^{0}\right]\right)$ des $\mathrm{Pa}$ rallelepipeds seinen größten Wert hat. Das ist dann der Fall, wenn die drei Einheitsvektoren $\mathfrak{r}_{1}{ }^{0}, \mathfrak{r}_{2}{ }^{0}, \mathfrak{r}_{3}{ }^{0}$ aufeinander senkrecht stehen. In diesen Richtungen werden sich bei der $\mathrm{NH}_{3}$-Bildung die drei $\mathrm{H}$-Atome an das N-Atom anlagern. Genau wịe beim $\mathrm{H}_{2} \mathrm{O}$ bewirkt die Abstoßung zwischen den $\mathrm{H}$-Atomen eine Spreizung der rechten Winkel zwischen den drei Richtungen, so daß die beobachtete nicht mehr genau rechtkantige Pyramidenform des $\mathrm{NH}_{3}$ entsteht.

Genau wie N verhalten sich die Elemente P, As, Sb, deren äußerste Schale dieselbe Elektronenkonfiguration wie $\mathrm{N}$ besitzt, nämlich drei spingleiche 
$p$-Elektronen. Es ist daher verständlich, daß Verbindungen wie $\mathrm{PH}_{3}, \mathrm{AsH}_{3}, \mathrm{PCl}_{3}, \mathrm{AsCl}_{3}, \mathrm{SbCl}_{3}$, $\mathrm{AsF}_{3}, \mathrm{AsBr}_{3}, \mathrm{AsJ}_{3}$ genau wie $\mathrm{NH}_{3}$ Pyramidengestalt besitzen mit dem P-bzw. As-bzw. Sb-Atom als Pyramidenspitze.

7. Die Eigenfunktion $\psi_{s p p}$. Unsere Theorie gibt auch die Erklärung dafür, warum in anderen Verbindungen wie $\mathrm{GaJ}_{3}, \mathrm{BF}_{3}, \mathrm{BCl}_{3}, \mathrm{AlBr}_{3}$ jeweils das erstgenannte Atom im Mittelpunkt und die an zweiter Stelle stehenden Atome an den Ecken eines gleichseitigen Dreiecks sitzen, daß diese Moleküle also im Gegensatz zum $\mathrm{NH}_{3}$ ebene Gestalt besitzen: Die Zentralatome ( $\mathrm{Ga}, \mathrm{B}, \mathrm{Al})$ besitzen in ihrer äußersten Schale zwei $s$-Elektronen (mit entgegen- gesetztem Spin) und ein $p$-Elektron, sind also zunächst als einwertig anzusehen. Da diese Atome aber in den oben genannten Verbindungen dreiwertig auftreten, muß man genau wie bei der $\mathrm{HgCl}_{2}$-Bildung annehmen, daß z. B. bei der Bildung des $\mathrm{GaJ}_{3}$-Moleküls infolge innermolekularer Kräfte eines der beiden $s$-Elektronen unter Spinumkehr in eine $p$-Bahn gebracht wird, so daß das Ga-Atom -im Molekülverband ein $s$ - und zwei$p$-Elektronen als Valenzelektronen besitzt. Nennt man $\mathfrak{a}$ und $\mathfrak{b}$ die (willkürlich zu denkenden) Achsenrichtungen der beiden $p$-Elektronen, so lautet die Eigenfunktion der so entstehenden dreiwertigen Elemente Ga, B, Al, nach $(1,1),(1,2),(1,3)$ :

$$
\begin{aligned}
\psi_{s p p}= & \left|\begin{array}{rrr}
g\left(r_{1}\right) & \left(\mathfrak{a} \mathfrak{r}_{1}{ }^{0}\right) \cdot f\left(r_{1}\right) & \left(\mathfrak{b} \mathfrak{r}_{1}{ }^{0}\right) \cdot f\left(r_{1}\right) \\
g\left(r_{2}\right) & \left(\mathfrak{a} \mathfrak{r}_{2}{ }^{0}\right) \cdot f\left(r_{2}\right) & \left(\mathfrak{b} \mathfrak{r}_{2}{ }^{0}\right) \cdot f\left(r_{2}\right) \\
g\left(r_{3}\right) & \left(\mathfrak{a} \mathfrak{r}_{3}{ }^{0}\right) \cdot f\left(r_{3}\right) & \left(\mathfrak{b} \mathfrak{r}_{3}{ }^{0}\right) \cdot f\left(r_{3}\right)
\end{array}\right| \\
= & g\left(r_{1}\right) f\left(r_{2}\right) f\left(r_{3}\right)\left([\mathfrak{a} \mathfrak{b}]\left[\mathfrak{r}_{2}{ }^{0} r_{3}{ }^{0}\right]\right)+g\left(r_{2}\right) f\left(r_{3}\right) f\left(r_{1}\right)\left([\mathfrak{a} \mathfrak{b}]\left[\mathfrak{r}_{3}{ }^{0} \mathfrak{r}_{1}{ }^{0}\right]\right) \\
& +g\left(r_{3}\right) f\left(r_{1}\right) f\left(r_{2}\right)\left([\mathfrak{a} \mathfrak{b}]\left[\mathfrak{r}_{1}{ }^{0} \mathfrak{r}_{2}{ }^{0}\right]\right) .
\end{aligned} \mid
$$

Wir denken uns auch jetzt wiede: elektronen auf einer Kugel $r_{1}=r_{2}=r_{3}=r$ beweglich. Dann wird, wenn die Abkürzung

$$
\mathfrak{x}=\left[\mathfrak{r}_{1}{ }^{0} \mathfrak{r}_{2}{ }^{0}\right]+\left[\mathfrak{r}_{2}{ }^{0} \mathfrak{r}_{3}{ }^{0}\right]+\left[\mathfrak{r}_{3}{ }^{0} \mathfrak{r}_{1}{ }^{0}\right]
$$

eingeführt wird:

$$
\psi_{s p p}=g(r) f^{2}(r)([\mathfrak{a} \mathfrak{b}] \mathfrak{z}) .
$$

Da $¥$ ersichtlich auch in der Form

$$
\mathfrak{k}=\left[\mathfrak{r}_{1}{ }^{0}-\mathfrak{r}_{2}{ }^{0}, \mathfrak{r}_{1}{ }^{0}-\mathfrak{r}_{3}{ }^{0}\right]
$$

geschirieben werden kann, so ist x ein Vektor, der senkrecht auf der Grundfläche der Pyramide steht,

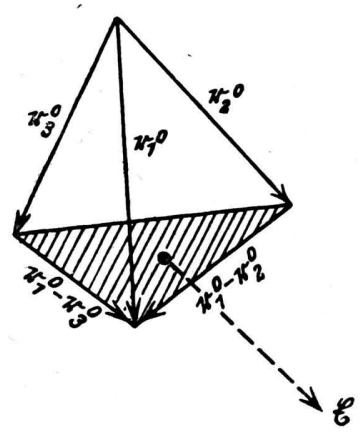

von deren Spitze die drei Einheitsvektoren $\mathfrak{r}_{1}{ }^{0}, \mathfrak{r}_{2}{ }^{0}$, $\mathrm{r}_{3}{ }^{0}$ ausgehen (siehe Abb.). Der Absolutbetrag des Vektors $\mathfrak{x}$ ist gleich dem doppelten Inhalt der Pyramiden-Grundfläche. Die Aufenthaltswahrscheinlichkeit $\left|\psi_{s p p}\right|^{2}$ nimmt nach $(1,10)$ dann ihren größten Wert an, wenn 1) $\mathfrak{x} \|[\mathfrak{a} \mathfrak{b}]$ ist und 2) diese Grundfläche |x́l ihren größten Wert besitzt. Das letztere ist dann der Fall, wenn diese drei Einheitsvektoren $\mathfrak{r}_{1}{ }^{0}, \mathfrak{r}_{2}{ }^{0}, \mathfrak{r}_{3}{ }^{0}$ in einer Ebene liegen und miteinander einen Winkel von $120^{\circ}$ einschließen. Dann ist die Pyramide auf ihre (gleichseitige) Grundfläche zusammengeschrumpft, und die drei Einheitsvektoren $\mathfrak{r}_{1}{ }^{0}, \mathfrak{r}_{2}{ }^{0}, \mathfrak{r}_{3}{ }^{0}$ weisen vom Mittelpunkt eines gleichseitigen Dreiecks nach dessen Ecken.

In diesen Richtungen der wahrscheinlichsten Elektronenverteilung werden sich dann andere einwertige Atome zur Molekülbildung anlagern. In diesen Richtungen sind also bei den zuletzt genannten dreiwertigen Elementen die Valenzstriche zu ziehen. Die Theorie liefert also auch hier dieselbe ebene Molekülstruktur wie die Erfahrung.

8. Die Eigenfunktion $\Psi_{s p p p}$. Der vierwertige Kohlenstoff, wie er z. B. im $\mathrm{CH}_{4}$ und in der Paraffinbindung auftritt, ist auf den ersten Blick nicht recht verständlich, weil das isolierte C-Atom nach spektroskopischen Untersuchungen zweiwertig ist. Das Kohlenstoffatom hat nämlich zwei der vier Elektronen seiner äußersten Schale mit antiparallelem Spin in $s$-Bahnen gebunden (= abgeschlossene Unterschale) und die restlichen beiden Elektronen als Valenzelektronen mit parallelem Spin in $p$-Bahnen. Um den vierwertigen Kohlenstoff, etwa im $\mathrm{CH}_{4}$, zu erklären, muß man nach $\mathrm{Pau}$ - 
ling annehmen, daß beim Heranbringen der $\mathrm{H}$-Atome infolge zwischenatomarer Kräfte das eine $s$-Elektron unter Spinumkehr in eine $p$-Bahn befördert wird, so daß beim $\mathrm{C}$ in den vierwertigen Verbindungen eines der Valenzelektronen ein
$s$-Elektron ist, die übrigen drei $p$-Elektronen sind.

Nennt man die Achsenrichtungen der drei $p$-Elektronen $\mathfrak{a}, \mathfrak{b}, \mathfrak{c}$, so lautet nach $(1,1),(1,2)$, $(1,3)$ die Eigenfunktion des vierwertigen Kohlenstoffs:

$$
\left.\begin{array}{rl}
\psi_{s p p p}= & \left|\begin{array}{llll}
g\left(r_{1}\right) & \left(\mathfrak{a} \mathfrak{r}_{1}{ }^{0}\right) \cdot f\left(r_{1}\right) & \left(\mathfrak{b} \mathfrak{r}_{1}{ }^{0}\right) \cdot f\left(r_{1}\right) & \left(\mathfrak{c} \mathfrak{r}_{1}{ }^{0}\right) \cdot f\left(r_{1}\right) \\
g\left(r_{2}\right) & \left(\mathfrak{a} \mathfrak{r}_{2}{ }^{0}\right) \cdot f\left(r_{2}\right) & \left(\mathfrak{b} \mathfrak{r}_{2}{ }^{0}\right) \cdot f\left(r_{2}\right) & \left(\mathfrak{c} \mathfrak{r}_{2}{ }^{0}\right) \cdot f\left(r_{2}\right) \\
g\left(r_{3}\right) & \left(\mathfrak{a} \mathfrak{r}_{3}{ }^{0}\right) \cdot f\left(r_{3}\right) & \left(\mathfrak{b} \mathfrak{r}_{3}{ }^{0}\right) \cdot f\left(r_{3}\right) & \left(\mathfrak{c} \mathfrak{r}_{3}{ }^{0}\right) \cdot f\left(r_{3}\right) \\
g\left(r_{4}\right) & \left(\mathfrak{a} \mathfrak{r}_{4}{ }^{0}\right) \cdot f\left(r_{4}\right) & \left(\mathfrak{b} \mathfrak{r}_{4}{ }^{0}\right) \cdot f\left(r_{4}\right) & \left(\mathfrak{c} \mathfrak{r}_{4}{ }^{0}\right) \cdot f\left(r_{4}\right)
\end{array}\right| \\
= & (\mathfrak{a}[\mathfrak{b} \mathfrak{c}]) \cdot f\left(r_{1}\right) \cdot\left(r_{2}\right) \cdot f\left(r_{3}\right) \cdot f\left(r_{4}\right) \cdot\left\{\frac{g\left(r_{1}\right)}{f\left(r_{1}\right)}\left(\mathfrak{r}_{2}{ }^{0}\left[\mathfrak{r}_{3}{ }^{0} \mathfrak{r}_{4}{ }^{0}\right]\right)\right. \\
& \left.-\frac{g\left(r_{2}\right)}{f\left(r_{2}\right)}\left(\mathfrak{r}_{3}{ }^{0}\left[\mathfrak{r}_{4}{ }^{0} \mathfrak{r}_{1}{ }^{0}\right]\right)+\frac{g\left(r_{3}\right)}{f\left(i_{3}\right)}\left(\mathfrak{r}_{4}{ }^{0}\left[\mathfrak{r}_{1}{ }^{0} \mathfrak{r}_{2}{ }^{0}\right]\right)-\frac{g\left(r_{4}\right)}{f\left(r_{4}\right)}\left(\mathfrak{r}_{1}{ }^{0}\left[\mathfrak{r}_{2}{ }^{0} \mathfrak{r}_{3}{ }^{0}\right]\right)\right\}
\end{array}\right\}
$$

Genau wie beim Stickstoff treten auch hier die Achsenvektoren $\mathfrak{a}, \mathfrak{b}, \mathfrak{c}$ nur in Form eines konstanten Faktors auf, der bei der Normierung der Funktion $\psi_{p p p}$ herausfällt. Denken wir uns auch hier die vier Valenzelektronen auf einer festen Kugel $r_{1}=r_{2}=r_{3}=r_{4}=r$ beweglich, so lautet $\psi_{s p p p}$ nach Fortlassung des unwesentlichen konstanten Faktors $(\mathfrak{a}[\mathfrak{b} \mathfrak{c}])$ :

$$
\psi_{s p p p}=f^{3}(r) g(r)\left\{\left(\mathfrak{r}_{2}{ }^{0}\left[\mathfrak{r}_{3}{ }^{0} \mathfrak{r}_{4}{ }^{0}\right]\right)-\left(\mathfrak{r}_{3}{ }^{0}\left[\mathfrak{r}_{4}{ }^{0} \mathfrak{r}_{1}{ }^{0}\right]\right)+\left(\mathfrak{r}_{4}{ }^{0}\left[\mathfrak{r}_{1}{ }^{0} \mathfrak{r}_{2}{ }^{0}\right]\right)-\left(\mathfrak{r}_{1}{ }^{0}\left[\mathfrak{r}_{2}{ }^{0} \mathfrak{r}_{3}{ }^{0}\right]\right)\right\}
$$

Jedes Glied der geschweiften Klammer ist das sechsfache Volumen der aus den betreffenden drei Einheitsvektoren gebildeten Pyramide. Die/geschweifte Klammer ist daher gleich dem sechsfachen Volumen des Tetraeders, dessen Ecken die Enden der vier Einheitsvektoren $\mathfrak{r}_{1}{ }^{0}, \mathfrak{r}_{2}{ }^{0}, \mathfrak{r}_{3}{ }^{0}, \mathfrak{r}_{4}{ }^{0}$ bilden. Die Aufenthaltswahrscheinlichkeit $\left|\psi_{s p p p}\right|^{2}$ hat also dann ihren größten Wert, wenn das Tetraedervolumen seinen größten Wert annimmt, also dann, wenn die Einheitsvektoren nach den vier Ecken eines regulären Tetraeders zeigen. In diesen Richtungen lagern sich bei der $\mathrm{CH}_{4}$-Bildung die vier H-Atome an. Die Wechselwirkung der vier $\mathrm{H}$-Atome untereinander ändert nichts mehr an der regulären Tetraederanordnung. Denn vier auf einer Kugel bewegliche, sich gegenseitig abstoßende H-Atome sind schon beim Fehlen des zentralen C-Atoms nur dann stabil angeordnet, wenn sie sich an den Ecken eines regulären Tetraeders befinden.

Aus den vorangegangenen Betrachtungen geht deutlich hervor, daß die tetraederartige Valenzbetätigung des Kohlenstoffs und allgemein die gewinkelte Valenz überhaupt eine Eigenschaft ist, die bereits im freien (C- bzw. N- bzw. O-) Atom ausgebildet ist und nicht von der Art der zu bindenden Atome, also von Kräften zwischen den gebundenen H-Atomen abhängt. Denn alle Aussagen über die Molekülgestalt konnten wir allein auf Grund der Eigenfunktion des Zentralatoms machen. Dafür spricht auch die Tatsache, daß z. B. bei Ersetzung zweier H-Atome des $\mathrm{CH}_{4}$ durch Cl-Atome das so entstandene $\mathrm{CH}_{2} \mathrm{Cl}_{2}$ trotz der Verschiedenheit der vier gebundenen Atome weitgehende Tetraedersymmetrie besitzt. Aus diesem Grunde sind von einem vierwertigen Kohlenstoffatom die Valenzstriche stets nach den vier Ecken eines regulären Tetraeders zu ziehen. Deshalb ist die Paraffinbindung (wo der Kohlenstoff vierwertig auftritt) geknickt, d.h. in den Paraffinen sind die C-Atome nicht geradlinig angeordnet, sondern in Form einer Zickzackkette mit dem Tetraederwinkel von $109^{\circ} 28^{\prime}$.

Genau wie der Kohlenstoff verhalten sich die Elemente Si, Ge, Sn, deren äußerste Schale denselben Elektronenaufbau wie der Kohlenstoff besitzt. Es ist daher verständlich, daß Verbindungen wie $\mathrm{SiCl}_{4}, \mathrm{GeCl}_{4}, \mathrm{SnCl}_{4}$ genau wie $\mathrm{CH}_{4}$ und $\mathrm{CCl}_{4}$ Tetraedersymmetrie besitzen.

9. Benzolring und Graphitbindung. Auf Grund der vorangegangenen Betrachtungen ist es einfach einzusehen, warum beim Benzolring und der Graphitbindung die C-Atome in Form eines ebenen Sechsecks angeordnet sind und keine Tetraederstruktur besitzen. Beim Benzolring und der Graphitbindung bewegen sich die Elektronen in einem 
periodischen Potentialfeld. Wie in der Elektronentheorie der Metalle gezeigt wird, können sich dann die Elektronen auf einem ,erlaubten Energieband“" infolge Tunneleffekts durch den ganzen (periodisch gebauten) Körper hindurchfressen. Und zwar hat, sofern der betreffende Stoff ein elektrischer Leiter ist, ein Elektron pro Atom die Eigenschaft, bedeutend lockerer am Atomrumpf gebunden zu sein als die übrigen, und bewirkt als Leitungselektron die elektrische Leitfähigkeit des betreffenden Stoffes. Diese Elektronen sind nach $\mathrm{Hü} \mathrm{ckel}^{5}$ nicht am einzelnen Atom lokalisierbar, sondern gehören dem gesamten Kristall bzw. Molekül an. Da die $p$-Elektronen lockerer am Atomrumpf gebunden sind als die $s$-Elektronen, so müssen wir annehmen, daß die nicht am einzelnen Kohlenstoffatom lokalisierbaren Leitungselektronen $p$-Elektronen sind. Dann verbleiben jedem vorher vierwertigen Kohlenstoffatom des Benzolrings noch ein $s$ - und zwei $p$-Elektronen als Valenzelektronen mit parallelem Spin. Diese bewirken nach $(1,10)$, daß das Valenzwinkelgerüst des so entstandenen dreiwertigen Kohlenstoffs (Eigenfunktion: $\psi_{s p p}$ ) aus drei vom Kohlenstoffkern ausgehenden Geraden besteht, die in einer Ebene liegen, und die miteinander einen Winkel von $120^{\circ}$ bilden.

Man erkennt sofort, daß mit diesem Valenzwinkelgerüst als einziger ebener Ring nur der Sechserring $\left(\mathrm{C}_{6} \mathrm{H}_{6}\right)$ möglich ist. Hiermit ist die quantenmechanische Begründung für das Auftreten

5 Z. Physik 70, 204 [1931]; 76, 628 [1932]. des ebenen Sechserrings bei den aromatischen Kohlenstoffverbindungen und der (dreiwertigen) Graphitbindung erbracht. In dieser Begründung für die besondere Stabilität des ebenen SechserRings unterscheiden wir uns grundlegend von Hückel, der die sechs im Ring nicht lokalisierbaren Elektronen als wesentlich für die Bindungsfestigkeit des Benzolrings ansieht. Auf eine nähere Diskussion der $\mathrm{Hückelschen} \mathrm{und} \mathrm{der} \mathrm{unserigen}$ Auffassung kann jedoch erst in der III. Mitteilg. eingegangen werden.

Die Tatsache, daß der Kohlenstoff dagegen im Diamantgitter vierwertig, also tetraedrisch auftritt, ist dadurch zu erklären, daß der Diamant im Gegensatz zum Graphit ein ausgezeichneter elektrischer Isolator ist. Das bedeutet: Im Diamant gibt das einzelne Kohlenstoffatom nicht wie beim Graphit ein Leitungselektron an den ganzen Kristall ab, sondern bleibt auch im Kristallgitter vierwertig, betätigt seine (vier) Valenzen also in den Tetraederrichtungen.

Hrn. Prof. W. Le n z bin ich für die Anregung zu dieser Arbeit zu Dank verpflichtet. Sie beruhte auf einer Kritik der Slater schen Theorie der gewinkelten Valenz. Es wurde von ihm die Notwendigkeit betont, die Valenzwinkel nicht auf die Orthogonalität von Eigenfunktionen zurückzuführen, sondern energetisch zu begründen, d. h. die Achsen-Vektoren $\mathfrak{a}, \mathfrak{b}, \mathfrak{c}$ nicht orthogonal zu nehmen, und die gewinkelte Molekülgestalt durch die Forderung kleinster Energie des gebundenen Zustands zu ermitteln. Die Antwort auf die hierin enthaltene Frage wird in der II. Mitteilg. erbracht und ist im wesentlichen in den obigen Formeln $\left(1,4^{\prime}\right),\left(1,5^{\prime}\right),(1,7),(1,10),(1,12)$ enthalten. 